\title{
Resource Aware Sensor-to-Actor Allocation Framework for WSANs Based on Voronoi Cells Theory
}

\author{
Sofia Maria Dima, Christos Antonopoulos, and Stavros Koubias \\ Department of Electrical and Computer Engineering, University of Patras, Patras, Greece \\ Correspondence should be addressed to Sofia Maria Dima; sdima@ece.upatras.gr \\ Received 30 October 2016; Revised 17 April 2017; Accepted 23 April 2017; Published 30 May 2017
}

Academic Editor: Jaime Lloret

Copyright (C) 2017 Sofia Maria Dima et al. This is an open access article distributed under the Creative Commons Attribution License, which permits unrestricted use, distribution, and reproduction in any medium, provided the original work is properly cited.

\begin{abstract}
Wireless sensor and actor networks (WSANs) have emerged as a promising research field and have been applied in a wide variety of application domains due to their capability of environment monitoring, event data processing, and decision-making by aiming at performing appropriate actions interacting with the environment. Coordination mechanisms among nodes and actors are a critical research challenge pertaining to the optimum allocation of sensors to a particular actor. Although efforts related to the node-to-actor coordination problem have been presented in the current literature, there is a significant oversight regarding critical characteristics such as the heterogeneous capabilities of the actors as well as the network's heterogeneous density. In this paper, aiming to address such shortcomings, we introduce the term Actor Service Capacity, which indicates the ability of an actor to serve a particular number of nodes. We also propose a novel node-to-actor coordination algorithm, based on the Voronoi tessellation, aiming to guarantee that the number of nodes, allocated to each actor, will not exceed its capabilities. Furthermore, a set of selection techniques are proposed so as to be applied on the coordination framework. Respective evaluation analysis offers useful conclusions and highlights the importance and the advantages of the proposed algorithm.
\end{abstract}

\section{Introduction}

Wireless sensor and actor networks (WSANs) have attracted active interest in recent years $[1,2]$ due to their ability to increase the effectiveness of numerous applications such as homeland security, battlefield reconnaissance, environmental monitoring rescue, and multirobots. A typical WSAN consists of a large set of sensor nodes (computing entities) reporting their data to significantly fewer actors (actuator) nodes, often denoted as controlling entities [3]. Sensors probe their surroundings and report their findings to one or multiple of actors, which process the collected sensor reports and respond to emerging events of interest.

From a hardware point of view, the sensor nodes are lowcost, low-power devices equipped with limited communication capabilities, while the actor nodes are usually mobile, more sophisticated, and powerful devices compared to the sensor nodes and are able to react in a detected event. The meaning of the term actor differs from the more conventional notion of actuator. An actuator $[1,4]$ is a device to convert an electrical control signal to a physical action and constitutes the mechanism by which an agent acts upon the physical environment. An actor, besides being able to act on the environment by means of one or several actuators, is also a network entity that performs networking and computational related functionalities, that is, receive, transmit, process, and relay data. For example, in the case of an environmental WSN application, where the main focus is the fire detection, the actors should be equipped with water sprinkler so as to react to the fire event.

Therefore, this kind of hybrid network (also denoted as cyber physical system [5]) extends typical WSNs goals of environment observation, event data processing, and decision-making by aiming at performing appropriate actions interacting with the environment [6]. In this context, the collaborative operation of sensors enables the distributed sensing of a physical phenomenon. After sensors detect an event that has occurred in the environment, the event data is distributively processed and transmitted to the actors, which 
gather, process, and reconstruct the characteristics of the event and eventually react to it [7]. WSANs can be seen as a distributed control system designed to timely react to sensor information with an effective action [8].

The process of establishing data paths between sensors and actors is referred to as sensor actor coordination [9, 10]. Unlike conventional Wireless Sensor Networks (WSNs), sensor and actor nodes must work hand in hand to collect and forward data and act on any sensed data collaboratively, promptly, and reliably. Hence, sensor actor coordination is essential when striving to find an optimal way to assign a set of sensors in a WSAN topology to a set of actors, considering actors' coverage range, sensors transmission ranges, energy, and time-constrained and computational requirements. Through this allocation, data paths between sensors and actor nodes are created [11], which are of paramount importance so as to coordinate sensing tasks, as well as acting on the physical environment based on the monitoring parameters. In addition, the scheduling and the management of the sensors nodes are also feasible through the sensorto-actor coordination. Finally, the restructure of the sensoractor assignment scenario can also occur through sensoractor coordination for reasons of fairness or unexpectable situations (actor failure). Once the event has been detected, the actors coordinate to reconstruct it, to estimate its characteristics, and to make a collaborative decision on how to perform the most appropriate reaction to the event. This process is referred to as actor-actor coordination [9]. As a result, the operation of a WSAN can be thought of as an event-sensing, communication, decision, and acting loop. For this reason, time-constrained communication is an important concern in WSANs to guarantee the timely execution of correct actions [12]. The energy efficiency of network communications is also crucial [13], since sensors typically are resource-constrained nodes with a limited battery lifetime. Furthermore, sensor network protocols and algorithms should be scalable and localized, as the number of nodes can be arbitrarily high.

Sensor-to-actor coordination is of utmost importance in WSANs, as there is no centralized control [14]. Sensors and actors need to coordinate with each other in order to collect information and take decisions on the next actions [1, 15]. Akyildiz et al. have described the main issues of the sensoractor coordination:

(i) What are the requirements of this communication?

(ii) Which sensors communicate with which actors?

(iii) How is this communication realized?

Towards meeting respective requirements, a critical challenge is guaranteeing optimal allocation of sensors to relative actuators [16]. In this context, relative efforts [17] rely solely on proximity parameter of sensors to actuating devices. However, as it is will discussed at the next section, such approach is quite myopic overlooking other critical actuators' parameters such as utilization rate, capabilities, and resource availability [18]. Such oversights lead to actuators overutilization and underutilization as well as suboptimal sensors semantic correlation, degrading reaction capabilities of the system as a whole. Driven by such observations as well as techniques used in mobile wireless networks defining cell tower capacity [19], we introduce a new term, called "Actor Service Capacity" (ASC), which quantifies the capability of an actor to service a subset of nodes. This capability depends on actor's remaining energy, computational power, service rate, and so forth. Thus, ASC is a crucial parameter that should be taken into account while partitioning the monitoring region into a number of cells equal to the number of actors.

To the best of our knowledge, this is the first work which focuses on allocating a set of sensors to a set of actors, assuring that the ASC criterion is satisfied. In current literature, there are research efforts [20] that propose techniques, which split the monitoring topology into regions, where the most powerful actor claims the biggest region. Although these techniques try to propose a fair scenario for scheduling sensors to actors, there is no guarantee that the ASC criterion is respected as well.

Given the above requirements, we propose a sensoractor coordination mechanism in the framework of Voronoi cells [21], which provides a scheduling methodology enabling optimal sensors correlation to specific actors, taking into consideration the proximity to the actors as well as the ASC. More specifically, we propose a novel clustering scheme, based on the Voronoi cells, in order to assign the sensors to a specific set of actors, which are characterized by a specific service capacity. The monitoring area is segmented into cells, each of which has a unique actor that is responsible for the nodes within the cell.

In summary, the contribution of this paper is threefold. Firstly, to the best of our knowledge, it is the first attempt that introduces the term of "ASC," enclosing concepts such as actor's computational power, actor's remaining energy, and actor's load backlog essential for emerging reaction to a detected event. In that respect, ASC and actor's distance from a specific node are the two criteria used in order to determine if the examined sensor is allocated or not to an actor. Secondly, Voronoi cells' theory is explored as an adequate theoretical substrate in order to implement a sensor-to-actor coordination algorithmic framework aiming to schedule the nodes within the monitoring area to the actor nodes. Both aforementioned criteria are taken as input to the coordination algorithm, which results in an assignment scenario, where each actor has under its responsibility a number of nodes less than or equal to its service capacity. Thirdly, a comprehensive study and evaluation effort are presented, concerning the assignment of sensors to actors, following different selection techniques within the Voronoi segmentation.

The remainder of this paper is organized as follows. In Section 2, relevant works are presented. In Sections 3 and 4, an introduction to the Voronoi diagrams is presented and the problem of this work is stated. In Section 5, the proposed algorithmic framework is investigated, while in Section 6 the holistic framework for evaluating the aforementioned concept as well as the performance analysis of our work is analyzed. Finally, in Section 7, the conclusions of our work are presented. 


\section{Related Work}

In order to better highlight the correlation and most importantly the enhancements of our work with respect to relative efforts specifically focusing on wireless sensor and actor networks, this section is structured according to two basic aspects: the application of the Voronoi theory in WSN or WSAN infrastructures and the problem of the sensor-toactor coordination in WSANs.

In the current literature, Voronoi frameworks are utilized to tackle various objectives in WSN environments. In [22], the authors propose a Voronoi-based schema that can be used in the management architecture of a WSN. More specifically, in case of a network with high density of sensor nodes, certain problems may arise such as the intersection of sensing area, redundant data, communication interference, and energy waste. A management application is necessary so as to achieve maximum network resources utilization. However, a highdensity network can introduce a fault-tolerant mechanism, increase precision, and provide multiresolution data. The network density control depends on the application. Hence, the authors propose a method to determine which nodes should be turned off or on. The management may take the sensor node out of service temporally. The authors propose a design using a Voronoi diagram, which decomposes the space into regions around each node.

Furthermore, in [23], the area coverage problem in directional sensor networks through rotatable orientation for each sensor is investigated. The authors propose the optimal coverage in directional sensor networks (OCDSN) problem, aiming to maximize coverage area while activating as few sensors as possible. The OCDSN is proven to be an NP-complete problem and the authors propose the greedy approximation algorithm based on the boundary Voronoi diagram.

In the same context, study [24] addresses the problem of space-partitioning in heterogeneous sensor networks, where the nodes have uniform symmetric sensing patterns, although their maximum distance differs. Emphasis is given in the inadequacy of classical spatial Voronoi tessellation for coverage purposes, compared to the proposed spacepartitioning technique, which takes into account this heterogeneity. The latter's definition is reflected in a way that the assigned regions are convex sets (contrary to weighted Voronoi diagrams); their construction is computationally efficient. Additionally, specific properties of Voronoi diagrams, which hold for homogeneous networks, are kept active. The proposed Voronoi definition degenerates into the classical one when the sensing radii of the nodes are equal.

In [25], a new approach related to Voronoi-based genetic clustering (VBGC) algorithm is proposed for enhanced energy efficient data aggregation. The authors claim that the proposed algorithm achieves energy efficiency by reducing the number of data transmissions in each round to cluster head and from it to base station (BS). The base station periodically executes the proposed algorithm to select new cluster heads after a certain period of time. Simulation results reveal that their proposed algorithm outperforms basic genetic algorithm.
With respect to the second aspects of this literature review, the problem of sensor-to-actor coordination has been investigated in several publications. The authors of [20] propose a sensor-actor coordination protocol for WSAN, based on Voronoi diagram and shortest path tree (VDSPT). Network nodes are organized into clusters based on weighted Voronoi diagram, where every cluster includes an actor and some sensors. The operation of VDSPT protocol is divided into rounds and the shortest path trees (SPTs) for routing are dynamically generated according to network states in each round. However, the proposed protocol takes into account geographical criteria and actor's remaining energy, while actor's capabilities are not considered. Consequently, although this work aims to offer a fair sensor-to-actor allocation strategy, as it assigns a larger region to actors with higher energy, it cannot guarantee that the number of nodes that will be assigned to an actor satisfies actor's ability to serve this number of nodes.

Imran et al. in $[4,26]$ propose Actor Connectivity Restoration algorithm focusing only on connectivity and coverage aspects so as to offer a solution in case of a failure of a critical actor node, which causes network partitioning into disjoint segments. The authors claim that ACR is designed so as to offer minimum recovery time and overhead, while minimizing the impact of recovery on coverage and connectivity. Two application-levels parameters are associated with each node: actor capability, related to actor coverage, and task criticality index, related to the priority of the current executed task. The two parameters are considered in order to find the critical nodes in the ACR algorithm. Similarly, Haider et al. [27] examine the problem of restoring interactor connectivity when a critical actor node fails. The term of sensor-actor reachability is relevant to actor coverage ("having sensors reachable to actors").

Finally, in [28], a sensor-actor coordination model is proposed based on an event-driven partitioning paradigm. Sensors are partitioned into different sets, and each set is constituted by a data-delivery tree associated with a different actor. The optimal solution for the partitioning strategy is determined by mathematical programming, and a distributed solution is proposed. In addition, a new model for the actor-actor coordination problem is introduced. The actor coordination is formulated as a task assignment optimization problem for a class of coordination problems in which the area to be acted upon needs to be optimally split among different actors.

Based on the highlighted relative effort, the proposed framework in this paper aims to enhance and extend relative efforts and tackle respective identified shortcomings or/and omissions. In the context of the proposed framework, the two aforementioned aspects are merged in a Voronoi-based sensor-actor coordination framework, where geographical criteria and ASC are the two major objectives which are going to be satisfied. Specifically, assuming a WSAN deployment where the sum of all ASC is greater than or equal to the total number of sensor nodes, an initial partition of the monitoring area is achieved by applying the classical algorithms of Euclidean, Additively Weighted Voronoi (AWV), and Multiplicative Weighted Voronoi (MWV) diagrams. As 
a result, the area is organized in clusters (Voronoi cell); each of them allocated a single actor, which serves as the cluster head, within the cell. On one hand, the Euclidean distance completely overlooks the ASC. On the other hand, the weighted distance (AWV and MWV) guarantees that an actor with a higher service capacity than another actor will claim a wider region. However, the latter implementation does not guarantee that the number of nodes within a Voronoi cell is less than or equal to their ASC. Thus, we propose a set of selection techniques which are applied upon the initial Voronoi segmentation. The final assignment may require more than one iteration loop until all sensors are assigned to an actor. The application of the selection techniques is crucial in scenarios where the nodes density is quite high around specific actors, avoiding nodes service starvation. It serves as an aging technique, trying to preserve nodes' service. In addition, the satisfaction of the ASC assures soft realtime requirements and prolongs application lifetime, as the workload posed on an actor is not exceeding its capabilities.

\section{Problem Formulation}

Given a specific network topology, consisting of a set of sensor and actor nodes, the main objectives of sensor-toactor coordination are the identification of optimal data paths between sensors and actors, the coordination of the sensing tasks, and the acting on the monitored environment. Aiming to minimize redundant transmissions, a critical requirement of relative algorithms is to preferably select paths so that sensor's data are transmitted only to one actor. The design of data paths can be envisioned as a clustering problem, where the networking area should be partitioned into clusters, while one actor will be present in each cluster and will be responsible for gathering the information from sensors nodes and react on specific events [29]. Hence, sensor nodes are scheduled/allocated to a specific actor [30]. A typical clustering scheme is based on geographical criteria so as to minimize the numbers of transmissions, aiming to optimize energy efficiency and network lifetime. However, these schemes do not consider actor's characteristics and capabilities such as the capability of an actor to react to a specific event reported by a sensor node pertaining to the semantic correlation between the actor and the physical event, the actor's backlog, the action range of the specific actors, and the processing power of each actor required to process the data and trigger the specific action scenario. The limitations posed by WSANs on all aforementioned aspects raise the necessity of novel approaches regarding coordination schemes that effectively extend typical metrics (e.g., geographical position) by incorporating real actors' capabilities and limitations.

Aiming to address these requirements, we introduce the definition of Actor Service Capacity (ASC), which can be envisioned as an analogy to the capacity of the cell towers in case of cellular networks. It is used as a generic term aiming to quantify the actor's capabilities. For example, if a sensor reading implies the execution of a classification algorithm, the ASC can be considered as the number of processing threads that can be executed efficiently on the MCU of the specific actor. From a network design point of view, the service capacity can be defined as the number of semantically correlated sensors that the actor is capable to serve at the same time. The kind of service varies from processing tasks to actuation tasks. Consequently, and very important to emphasize, Actor Service Capacity can enclose concepts such as actor's computational power, actor's remaining energy, and actor's load backlog which is critical for prompt and accurate reaction to a detected event. It is a way to compare heterogeneous wireless actor platforms in terms of their hardware characteristics (processors, memory, and network bandwidth capabilities). In this context, it effectively comprises a novel term aiming to quantify the ability of an actor to serve efficiently a specific number of nodes. Hence, assuming an actor that is characterized by ASC equal to 30, it means that this actor can serve up to 30 nodes concurrently.

We assume that each actor is characterized by a specific service capacity. The sum of all ASC is greater or equal to the number of nodes within the WSAN monitoring area. Hence, the problem is to find an allocation mechanism in order to assign the sensors to the actors, assuring that actors' service capacity is not exceeded and that actors' resources are fairly allocated to the sensor nodes.

Based on the described problem formulation, the Voronoi cells comprise a valuable tool which is extended so as to achieve optimal region partitioning. Specifically, the native consideration of Voronoi cells does not take into consideration ASC. Hence, we provide a complete algorithmic framework, based on Voronoi cells, so as to give an efficient solution to the described problem.

3.1. Overview of Voronoi Cells. Consider a set of points, $P=$ $\left\{p_{1}, p_{2}, \ldots, p_{n}\right\}, p_{i} \in R \times R$, which are called particles or sites. The Voronoi cell of a particle $p_{i}$ is a convex set of points, which is defined as the locus $\left(\Phi_{i}\right)$ of points (1), which are closer to the $p_{i}$ point rather than any other particle $p_{j}$ of the set $P$.

$$
\Phi_{i}=\left\{x \in X \mid\left\|x-p_{i}\right\|<\left\|x-p_{j}\right\|, i \neq j\right\} .
$$

Hence, the cells define a partition of $2 \mathrm{D}$ space which is called Voronoi partitioning or Dirichlet partitioning [31]. The common limits between the Voronoi cells are called Voronoi edges and indicate the points that are equidistant from two sites. The Voronoi vertices indicate the points that are equidistant from three or more sites. The Voronoi vertices, as well as the Voronoi edges, constitute the skeleton of the Voronoi Diagram, as shown in Figure 1. This represents the classic Voronoi diagrams, which are based on the Euclidean distance.

However, in current literature, Additively Weight Voronoi (AWV) [32] and Multiplicative Weighted Voronoi (MWV) [33] diagrams have been also investigated. In both cases, the distance of a point to a specific site is the weighted Euclidean distance. More specifically, each site $\left(p_{i}\right)$ is characterized by a weight factor $w_{i}$. In case of AWV, the distance is defined as

$$
d\left(x, p_{i}\right)=\left\|x-p_{i}\right\|-w_{i}
$$




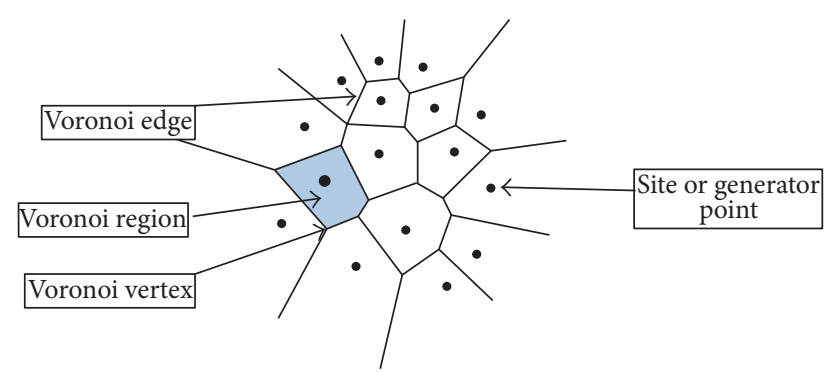

FIgURE 1: Voronoi diagram.

while in case of MWV the distance is

$$
d\left(x, p_{i}\right)=\frac{\left\|x-p_{i}\right\|}{w_{i}} .
$$

\section{Proposed Technique}

Given a monitoring $2 D$ area, which consisted of a set of actors $A=a_{1}, a_{2}, \ldots, a_{m}$ and a set of sensors $S=s_{1}, s_{2}, \ldots, s_{n}$, the problem is to correlate the sensors to actors. As the radio interface is the most energy-consuming component in a typical WSN platform, the distance between the sensor and its actor should be minimum. Hence, the application of the Euclidean distance seems to offer the best solution. More specifically, the Euclidean distance between the sensor node and the actor (site) is calculated for each sensor node and for all actors. The minimum distance of each sensor indicates the actor that the sensor should be allocated to.

However, this consideration does not take into account actors' limitations such as residual energy, action range, and service capacity. We are going to investigate how the ASC can be satisfied. AWV and MWV can provide a solution considering ASC as the site's weight factor. Following this approach, the weighted distance between the sensor node and the actor (site) is calculated for each sensor node for all actors. The minimum weighted distance of each sensor indicates the actor that the sensor should be allocated to. Although native AWV and MWV approaches do take into consideration actors' capacity in a relatively abstract way, they fail to optimally exploit respective requirement so as to guarantee avoidance of overutilization or underutilization. For example, considering three actors with indicative service capacities equal to 15,21 , and 24 , respectively, native $\mathrm{AWN}$ and $\mathrm{MWN}$ may lead to utilization degree at the same ascending order but at the same time not avoiding cases of overutilization (e.g., of the actor with highest capacity) and/or underutilization (e.g., of the actor with lowest capacity). However, the number of nodes that are inside each Voronoi cell can exceed the respective ASC. This is due to the fact that the Voronoi cell does not take into account the area's density.

Assuming that the sum of ASC is greater or equal to the total number of sensor nodes, the problem can be defined as how to schedule nodes to actors. We propose four selection techniques, which can be applied on the Voronoi algorithm, so as to assure ASC:

(i) Random: the selection is based on a random generator (ii) Distance: the nodes are sorted based on the proximity to the actor, and the sensors that are closer to the actor are selected

(iii) Tensor: a linear interpolation function is applied:

$$
f(\text { tensor })=\text { tensor } * p+(1-\text { tensor }) * \text { rand. }
$$

As it can be easily observed, the tensor value can increase or decrease the randomness. More specifically, when the tensor has a value of $0, f$ (tensor) $=$ rand, while the tensor is equal to one $f$ (tensor) $=p$. The variable $p$ represents the probability that a sensor is selected, which has been extracted according to the following equation:

$$
p(\text { sensor })=\frac{d(\text { sensor }, \text { site })}{\max _{d}}
$$

(iv) Rounds: each actor selects the closer sensor at odd round and a far one at even round

Initially, each sensor determines the actor to which it is allocated based on the minimum (weighted or not) distance (Algorithm 1). Hence, an initial assignment is achieved and each actor is aware of the nodes residing in its region. Following this phase, each actor checks if the number of sensors that belong to each cell exceeds its service capacity. If not, then all sensors' assignments are approved. Otherwise, a selection technique is executed so as to select the nodes (number of nodes $=$ ASC) that will be assigned to the specific actor (Algorithm 2). The rest of the sensors are considered unassigned in this iteration of the proposed technique. After all actors have completed the first iteration, the numbers of unassigned sensors and the actors which have not exceeded their service capacity are known. Thus, a second iteration considering solely actors with available capacity as sites is needed. After the Voronoi partitioning has taken place, the selection technique is applied again if needed. The iteration loop terminates, when all nodes are scheduled to a specific actor (Figure 2).

At this point, it is worth noting that the proposed techniques assume global knowledge of the WSAN topology (location sensors and actors). In addition, ASC, which is also additional info on the monitored WSAN topology, is also known. Hence, the initial Voronoi tessellation as well as the final assignment of each sensor to the "optimal/suboptimal actor" after applying selection techniques on the initial Voronoi tessellation is performed by a centralized management entity as a preprocessing step. The algorithms output will be considered to the final firmware which is going to be deployed to the sensors/actor nodes.

\section{Simulation Framework}

Aiming to offer an objective evaluation, the proposed framework has been implemented under .Net Framework, using C\#. It must be noted that the evaluation results aim to offer insight into how the sensors are allocated to a specific set of actors, using the native algorithms of Voronoi splitting, 


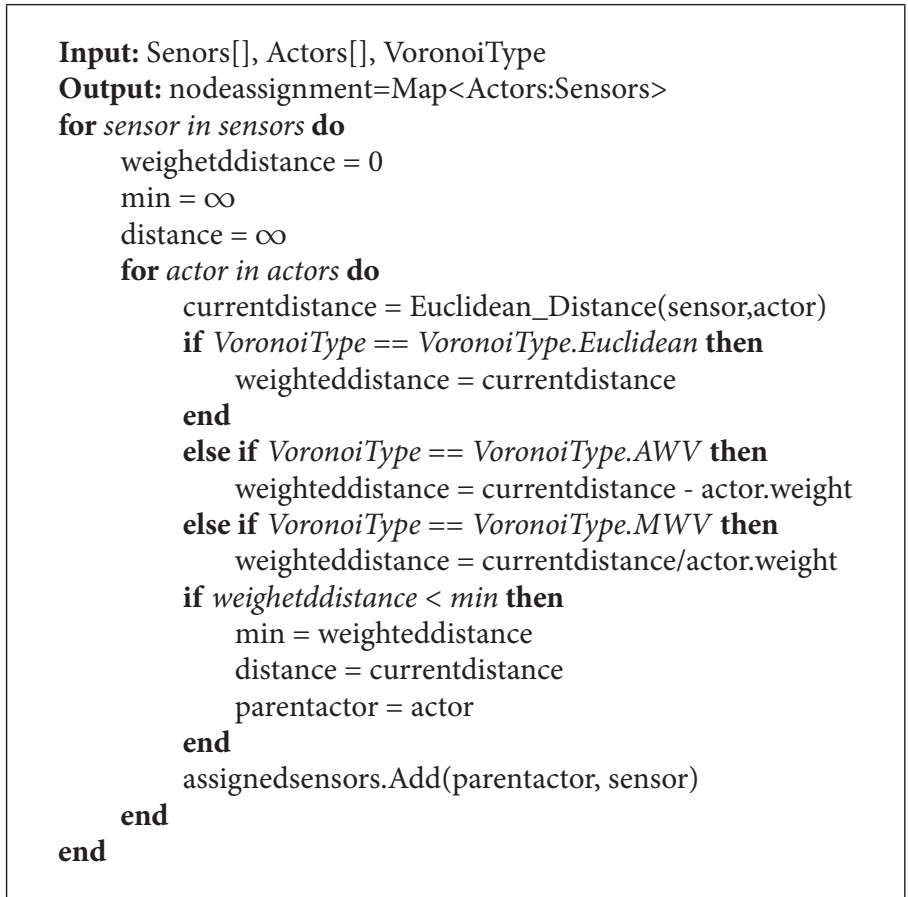

Algorithm 1: Voronoi splitting.

and how this schedule is affected by applying the presented techniques. As this work aims to provide a comparative study of the aforementioned techniques, a parameterized simulation framework has been set up. More specifically, a topology generator has been implemented so as to execute the proposed coordination algorithm for a high number of random topologies. This can offer more objective, repeatable, and verifiable results. For each topology, the Voronoi algorithms (Euclidean, AWV, and MWV) are applied. Hence, initial assignment of the sensors to the existing actors is achieved in a single iteration. However, ASC is not taken into account. Then, each one of the aforementioned selection techniques is applied so as to satisfy actors' capacity. In this case, the final assignment is achieved in more than one iteration. In each iteration, the Voronoi type and the selection technique are applied on the unassigned sensors. When all sensors have been assigned to an actor, the process terminates. At this point, it is worth noting that, for the Tensor technique, the tensor value varies from 0 to 1 , with 0.2 step. When the tensor has a value of 0 , the linear interpolation technique is identical to the Random selection technique, while when the tensor has a value of 1 it is identical to the Distance selection technique. The evaluation results have been extracted after simulating 150 WSAN topologies, and the average values were calculated.

Topology. A random topology generator has been implemented in C\#. The monitoring area is considered as a rectangle, characterized by its length and width. Both dimensions are characterized as $1200 \mathrm{~m} * 1200 \mathrm{~m}$. The topology's dimension has sense when considering absolute distances between sensor nodes and actors. In the constructed area, a set of 6 actors is created. The position of each actor within the WSAN area is random. In addition, each actor is characterized by a weight. Actually, the weight is equal to ASC. A set of 140 nodes is also scattered in the WSAN area.

ASC. In all scenarios, the total sum of actors is greater than or equal to the number of nodes. The ASC is the weight factor in the weighted distance, considered in the AWV and MWV Voronoi diagrams. Each actor has a different service capacity, allowing the modelling of the heterogeneity of the actor platforms as well as a more objective evaluation analysis. When the total capacity is equal to the number of nodes, the sensor-to-actor algorithm is quite stressed and there is a possibility of scheduling a node to a far way actor. This probability is reduced when the actors' capacity is larger than the sensors allowing them to more freely select nodes. In addition, when the total service capacity is greater than the number of nodes, a comparison of actors' utilization is of utmost importance. More specifically, ideally when the overall capacity is greater than the number of nodes which should be served, all actors should contribute to the final schedule in order to satisfy the criterion of fairness. Otherwise, network and computational resources are unexploited, and situations where actors are fully utilized (while at the same time there are other actors which have not been assigned any sensor) are possible.

Evaluation Metrics. After applying the Voronoi tessellation algorithm, without and with selection techniques, an assignment schedule has been extracted. For the above cases, we have extracted the following metrics:

(i) Average distance: in each topology the distance between a sensor and its corresponding actor is 


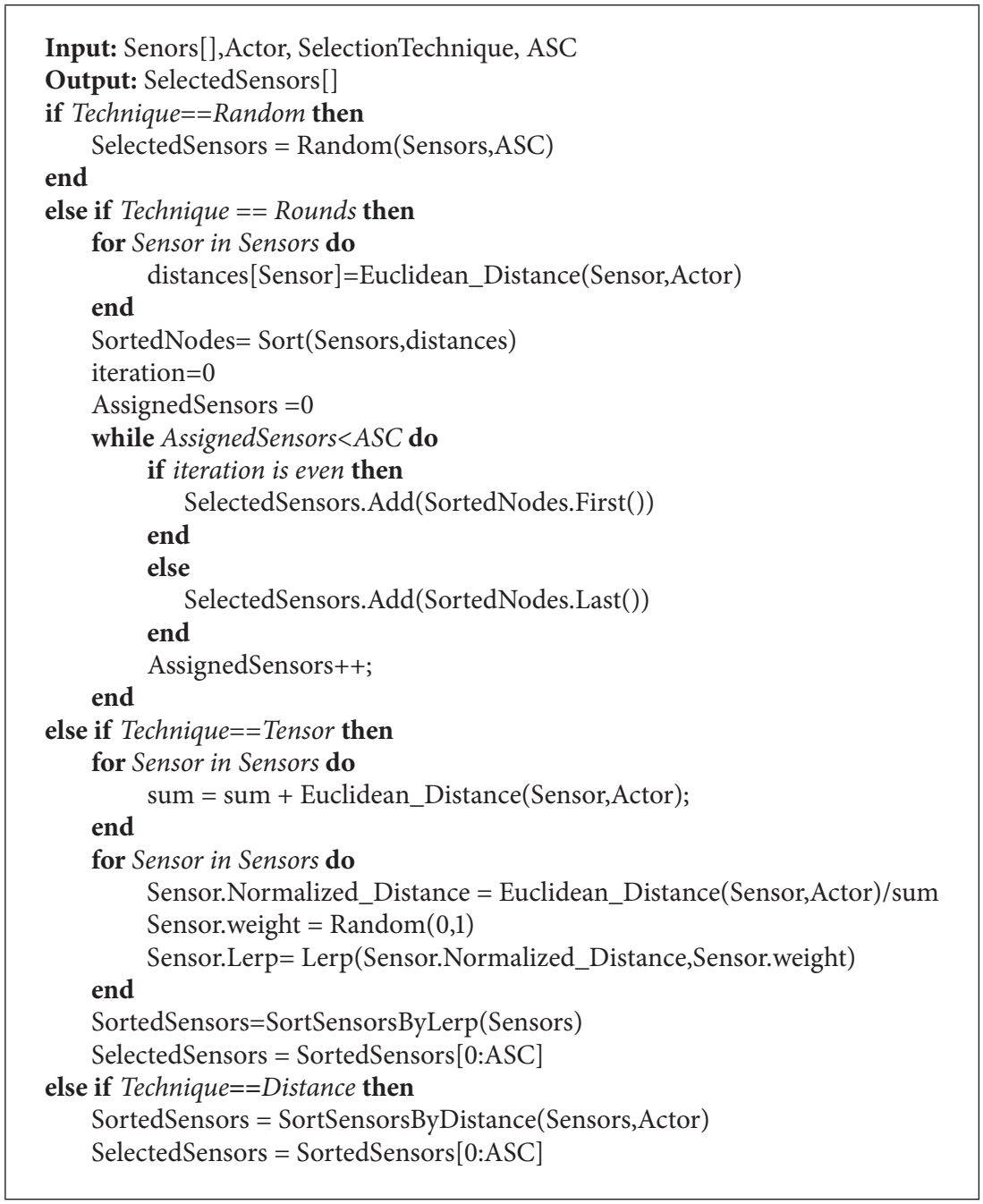

Algorithm 2: Selection techniques.

calculated. The mean value of these distances is extracted for each topology. The average value considering the 150 topologies used in our framework is considered as the average distance

(ii) Averaged maximum distance: in each topology the distance between a sensor and its corresponding actor is calculated. The max value of these distances is extracted for each topology. The average value considering the 150 topologies used in our framework is considered as the averaged maximum distance. Maximum distance is important to avoid misleading conclusions from solely average distance measurements caused in cases where a few maximum measurements exist, with emphatic deviation from the average distance, thus comprising potentially important bottlenecks

(iii) Deviation in average distance: the deviation of each selection technique from the reference technique ("None") is calculated for all the Voronoi types
(Euclidean, AWV, and MWV). The deviation of the average distance is extracted according to (6). It shows the average percentile difference (error) of the Voronoi splitting algorithm following a selection technique from the native consideration ("None"). It is noted that Euclidean Voronoi segmentation algorithm serves as reference point since by definition it provides the minimum distance metric. Therefore, it is critical to quantify the way native AWV and MWV approaches (i.e., without considering any selection technique) affect this metric taking into consideration actors' capacity. Furthermore, it is also of paramount importance for this work to objectively quantify the way respective measurements are affected by the proposed selection techniques trying to optimally utilize actors' capacity. Thus, this metric indicates the distance metrics performance fluctuation, considering a specific Voronoi type (Euclidean, AWV, and 


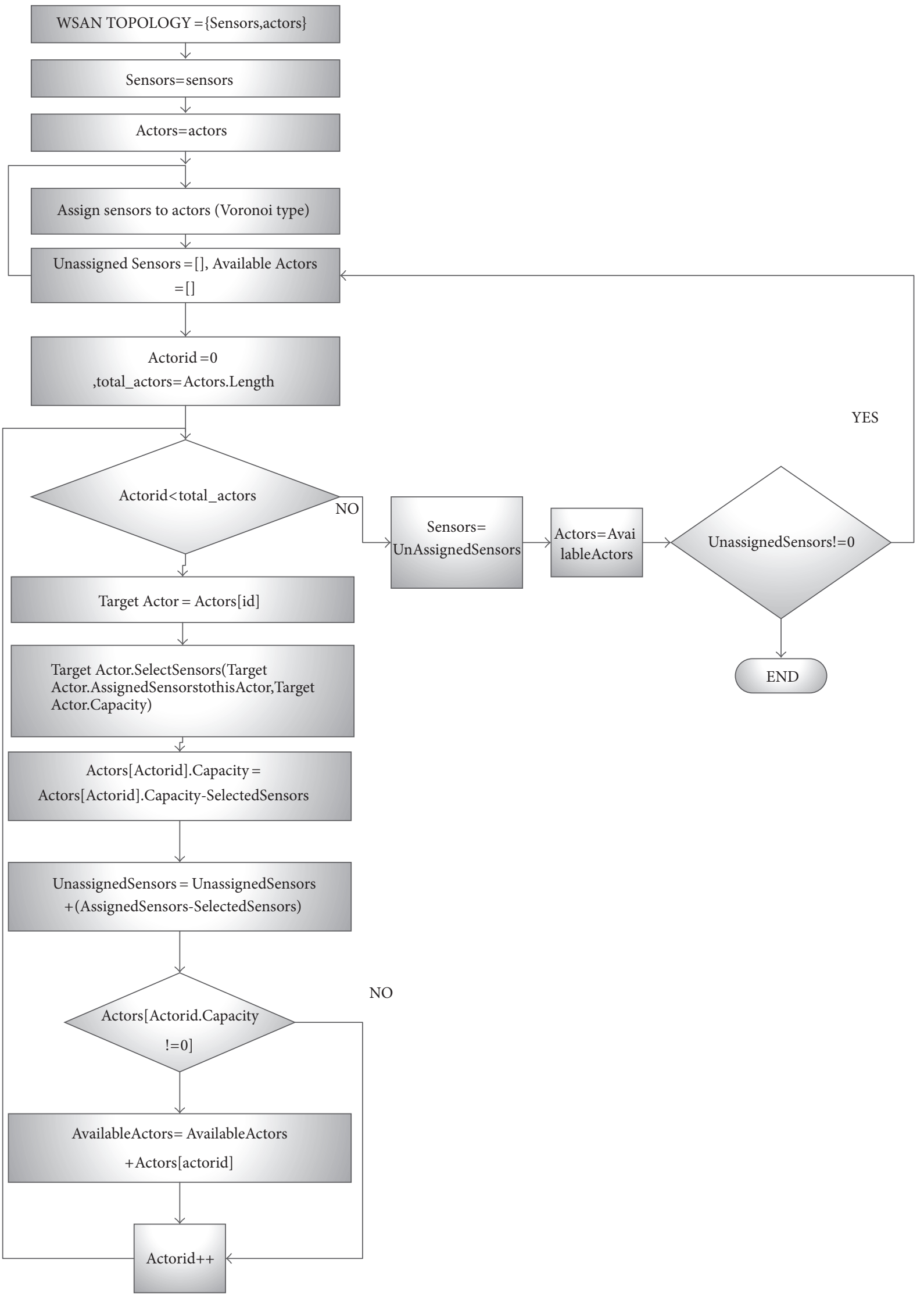

FIgure 2: Proposed technique. 
MWV) as well as the effect of a selection technique tuning the Voronoi type.

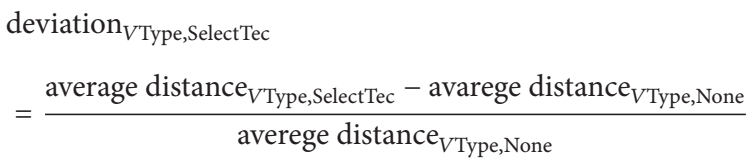

(iv) Deviation in averaged maximum distance: the deviation of the max average distance is extracted according to the following equation:

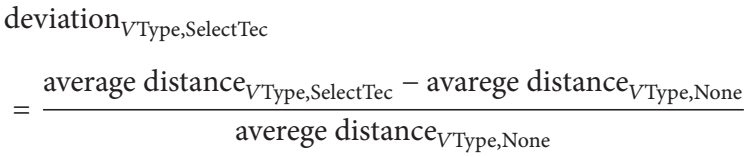

(v) Actors' utilization: in each experimental scenario, each actor is responsible for a set of sensors. Considering the case where the total service capacity is equal to the number of sensors, each actor should be $100 \%$ utilized. This is achieved when applying a selection technique. However, in case that "None" is followed (i.e., there is no selected technique-native consideration), an actor can be underutilized or overutilized. An actor is considered as underutilized when the number of nodes assigned to it is lower than its service capacity. The utilization percentage in this case is considered as a positive number:

utilization percentage

$$
=\frac{(\text { service capacity }- \text { assigned sensors }) * 100}{\text { service capacity }} .
$$

In contrast, an actor is considered as overutilized when the number of actors assigned to it is greater than its service capacity. The utilization percentage in this case is considered as a negative number:

utilization percentage

$$
=\frac{(\text { assigned sensors }- \text { service capacity }) * 100}{\text { service capacity }} \text {. }
$$

The average overutilization as well as underutilization percentage is extracted for each topology, while the mean value for the 150 simulated topologies is used. In case that the total service capacity is equal to the number of sensor nodes, the overutilization/underutilization percentage of the actors indicates the nonoptimal use of the actors' nodes. When the total service capacity is greater than the number of sensor nodes, a critical point is to investigate how fair is each technique regarding the management of the actors' resources

(vi) Algorithm's iterations: as described in Figure 2, in case the splitting is achieved following a selection technique, the final assignment will occur in more than one iteration. For each topology, the iterations required for the final assignment are calculated and the averaged value for the 150 topologies is used. This metric is quite significant in case this algorithm is ported on an embedded system pertaining to both the processing and the memory requirements.

\section{Evaluation Analysis}

6.1. Setup. In this section, the results of the experimental evaluation are presented focusing on the comparison of the native Voronoi splitting algorithms ("None") (Euclidean, $\mathrm{AWV}$, and MWV) and the Voronoi algorithms followed by the application of the selection techniques. (Figure 3) Our evaluation results are analyzed in two subsections. In the first one, the overall capacity is equal to the sensor nodes (e.g., 140), where the advantages of applying the selection techniques compared to not applying any selection technique are highlighted. More specifically, the native algorithms will conclude to a task assignment scenario, where a significant percentage of the actors are overutilized, while others are underutilized. At the same time, the coordination schemes incorporating a selection technique guarantee zero overutilization and zero underutilization. In addition, the selection techniques are investigated in a comprehensive way so as to reveal and offer insight regarding the respective advantages and disadvantages. In the second subsection, the above approaches are compared assuming total actors capacity equal to 220, while the number of nodes remains at 140 . The utilization of actors comprises a cornerstone evaluation metric, as its value indicated the importance of each selection technique, and it is a direct way to compare the selection techniques.

6.2. Total Actors Capacity Equal to the Number of Sensor Nodes. In Figure 4 the average distance in absolute values between a sensor and its corresponding actor is depicted, considering the 150 simulated topologies. As expected in all three Voronoi types considered, minimum geographical distance values are recorded when distance comprises the sole parameter considered; that is, no selection technique is applied. As extracted in Figure 4 from the "None" case, among the three approaches, Euclidean and AWN record the minimum and approximately equivalent performance. In contrast, MWV leads to substantially increased distances by approximately $37 \%$. This difference in the average distance when no selection technique is applied indicates that the Euclidean distance and the weighted distance in the case of the AWV algorithm lead to a splitting scenario, where the geographical criteria are the dominant. The weighted distance in the MWV algorithm can lead to a splitting scenario, where a sensor can be assigned to a far away actor because of its weight. Considering the "None" technique's measurements as reference values, by definition, all other techniques will exhibit increased distance measurements. This increase effectively represents the tradeoff aiming to optimally use actuators capacity and thus tackle the unfair and uneven overburden of actors when myopically focusing only on the distance factor. An interesting observation is that 


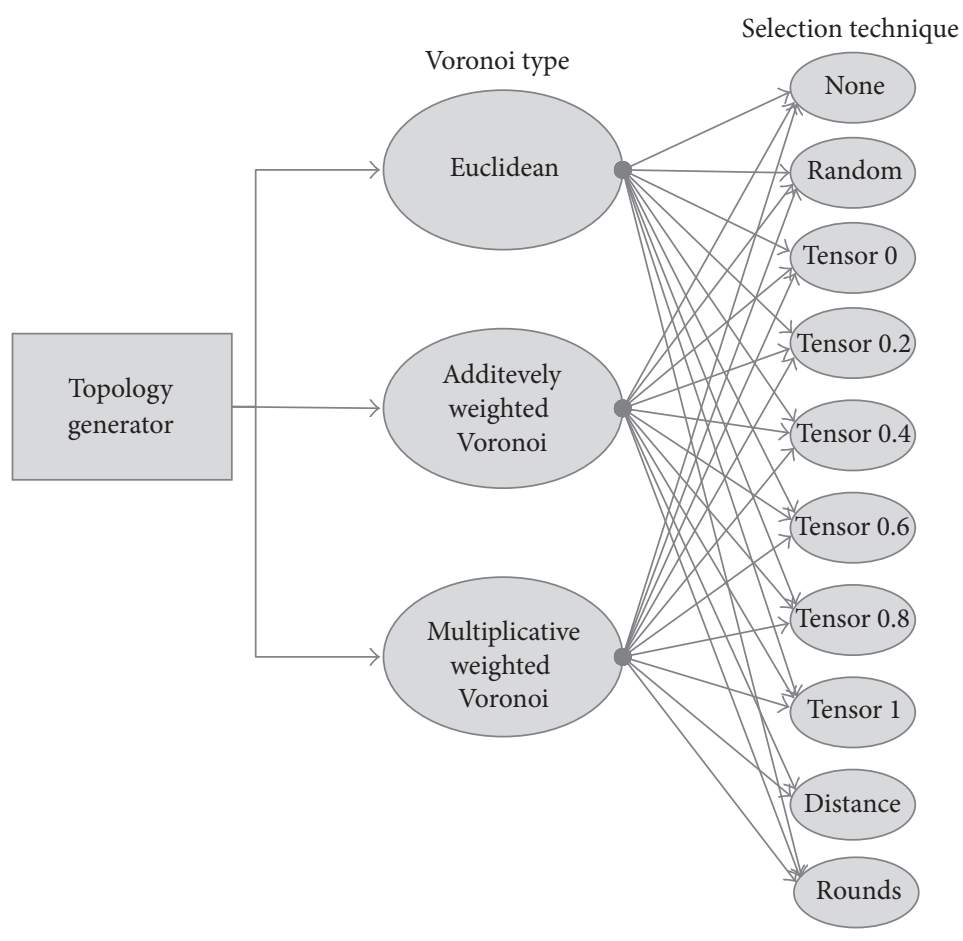

FIGURE 3: Simulation framework.

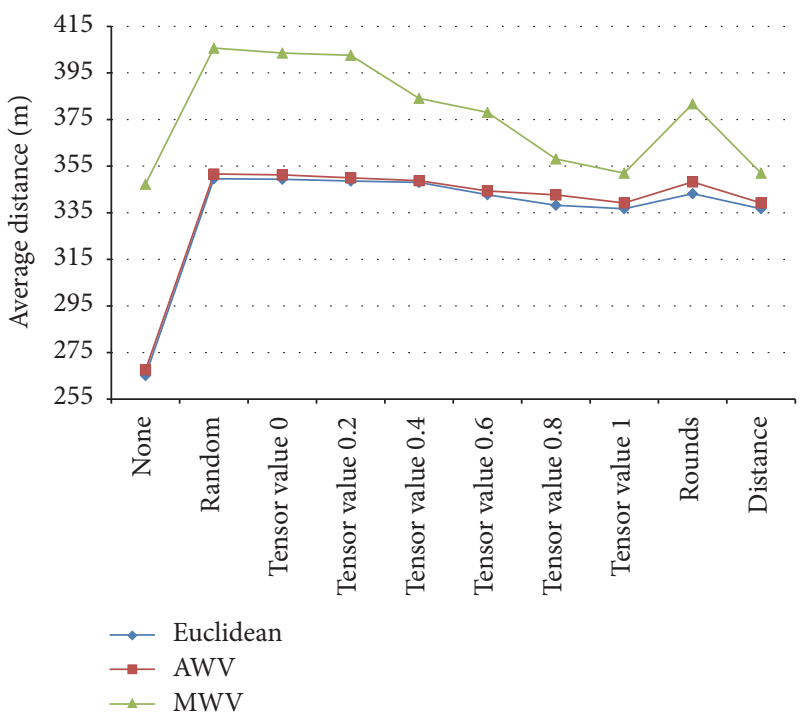

FIgURE 4: Average distance. Total capacity $=140$.

although MWV yields the higher distances, it also seems to be less affected by the considered techniques since absolute distance increase is significantly lower compared to Euclidean and AWV. Specifically, in worst case technique (i.e., Random), MWV leads to a 65-distance-units deviation, whereas, respectively, AWV and Euclidean yield an 85-distance-units deviation.

In Figure 5, the averaged maximum distance between a sensor and its corresponding actor is depicted. As explained in the previous Figure 4, the "None" selection technique

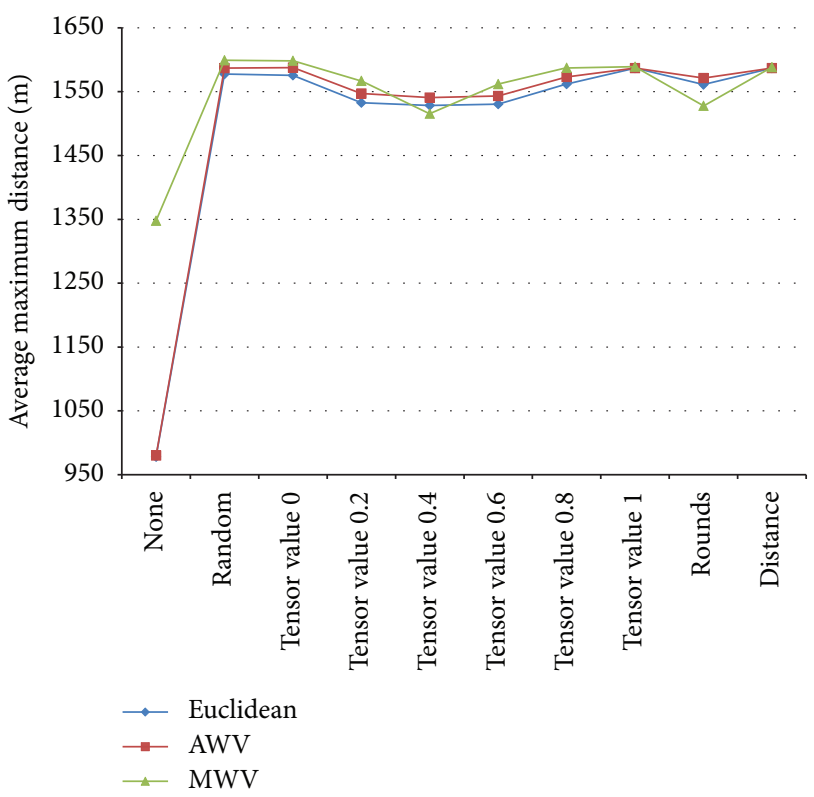

FIgURE 5: Average maximum distance. Total capacity $=140$.

is the reference point, and hence the average maximum distance has the lowest value. Once again Euclidean and AWV graphs have similar behaviors while tuning all the selection techniques. What is interesting here to observe is that, with respect to this metric, all three Voronoi types have similar behaviors with respect to the considered selection techniques. This can be very useful, since it indicates a bounded maximum distance approximately the same in all 


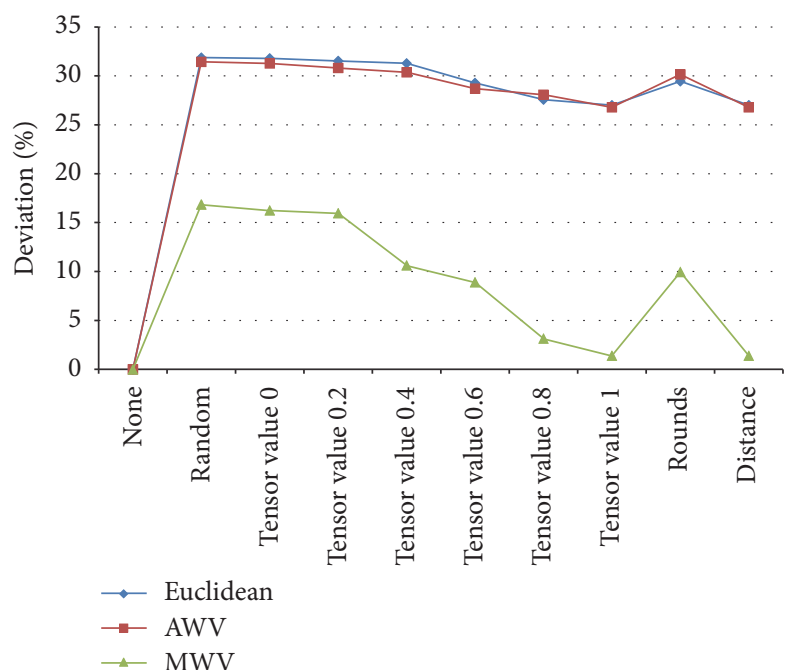

Figure 6: Deviation in average distance. Total capacity $=140$.

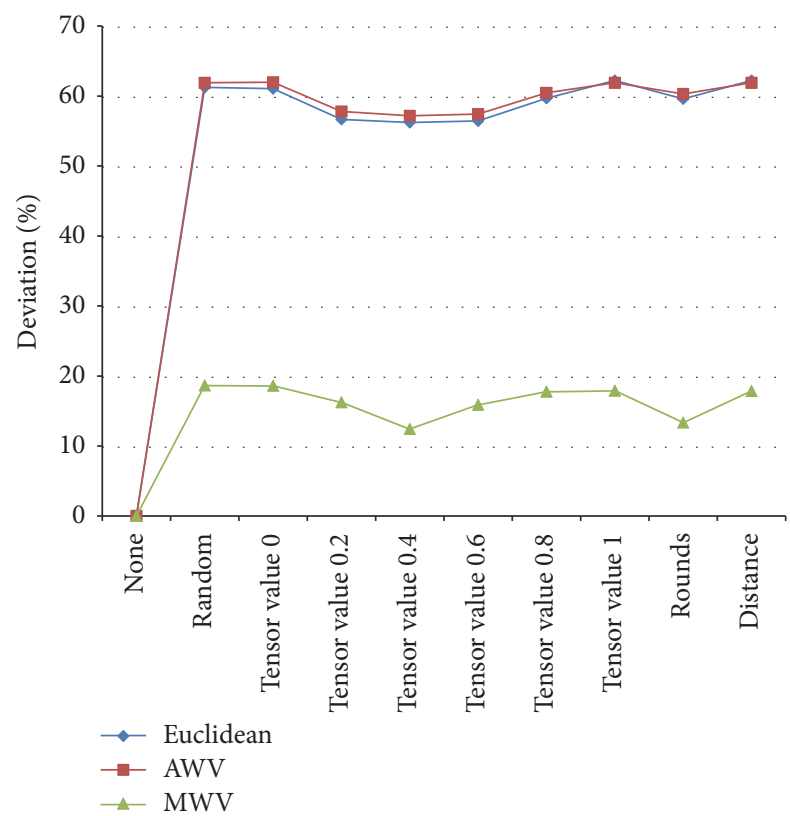

FIGURE 7: Deviation in max distance. Total capacity $=140$.

cases. So, in other words, this is not a performance parameter that is likely to favor the selection of any of the proposed techniques.

In Figures 6 and 7, the percentile deviation between the selection techniques and the "None" technique is presented for all the Voronoi types, offering valuable insight especially with respect to the tradeoff between optimal capacity utilization and distance overhead imposed. As it can be observed, the application of the selection techniques considering the MWV algorithm leads to a percentile deviation varying from $17 \%$ to $2 \%$. This is of utmost importance, as the application of the selection techniques guarantees the ASC criterion, while retaining the average distance between a sensor and its actor to a minimum. At the same time, the application of the

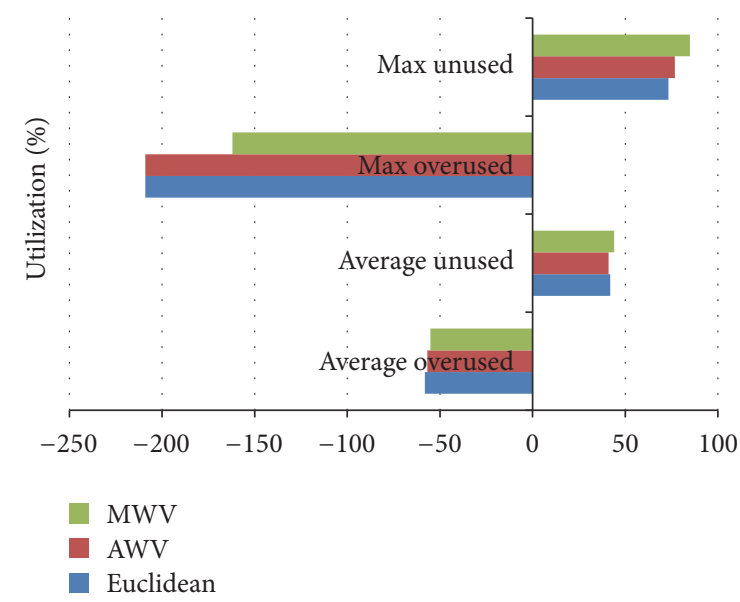

FIgURE 8: Actors utilization with no selection technique. Total capacity $=140$.

selection techniques on both Euclidean and AWV algorithms leads to a much more pronounced increase of the average distance which varies from $28 \%$ to $32 \%$. So it is highlighted that even though MWV exhibits the higher distances as a native technique, at the same time it possesses the least distance overhead when utilized. Particularly in cases like tensor value 1 and Distance techniques, this overhead can be considered even negligible. Therefore, if MWV distances are acceptable, then this Voronoi type is probably the best choice so as to guarantee minimum overhead. However, in case absolute distance is a critical requirement to a specific scenario, Euclidean and AWV Voronoi types offer critical advantages.

The percentile deviation in the maximum average distance is shown in Figure 7. Once again the lowest deviation from the reference technique is offered by the MWV type. The deviation varies from $13 \%$ to $18 \%$, with the lowest values on the Tensor technique with tensor value equal to 0.4 and on the Rounds technique. The deviation considering the Euclidean and the AWV types exceeds the value of $60 \%$, while the lowest values are offered by the Tensor techniques. What is verified through these measurements is that although Euclidean and AWV Voronoi types offer the minimum absolute values, MWV Voronoi types seem to be significantly less affected by the selection techniques considered in this paper.

As indicated, the main objective of applying a selection technique is to address the actors misuse with respect to their limited capacity regarding allocated sensors. Therefore, in this paragraph, we will highlight the significant benefits offered by the proposed selection techniques from two different approaches. On one hand, it is emphasized that, by integrating any of the proposed selection techniques in the native Voronoi algorithm, we are able to achieve optimum actor utilization in all cases. Considering that the aggregate sensors are equal to the aggregate actors capacity, this implies yielding $100 \%$ utilization of each actor, $0 \%$ overutilization, and $0 \%$ underutilization. This performance is effectively the main advantage of the proposed techniques. On the other hand, authors quantified the anticipated misuse of actors assuming native Voronoi algorithm utilization. Respective quantification measurements are depicted in Figure 8. For 
TABLE 1: Average iterations. Total ASC $=140$.

\begin{tabular}{lcccccccccc}
\hline & None & Random & Tensor 0 & Tensor 0.2 & Tensor 0.4 & Tensor 0.6 & Tensor 0.8 & Tensor 1 & Rounds & Distance \\
\hline Euclidean & 0 & 3.59 & 3.57 & 3.49 & 3.48 & 3.46 & 3.53 & 3.53 & 3.57 & 3.53 \\
AWV & 0 & 3.53 & 3.57 & 3.53 & 3.47 & 3.46 & 3.49 & 3.51 & 3.59 & 3.51 \\
MWV & 0 & 4.01 & 3.99 & 3.92 & 3.97 & 4.04 & 4.02 & 4.01 & 4.03 & 4.01 \\
\hline
\end{tabular}

reasons of presentation and in order to clearly distinct the two cases, underutilization recordings are presented with positive percentage values and respective overutilization recording with negative percentage values. Specifically, as easily extracted, when applying the raw Voronoi algorithm, the final assignment deterministically leads to a high percentage of actors that are responsible for a number of sensors corresponding to a capacity higher than that of the actor's or to actors that have their power underutilized, allocating a number of sensors lower than their service capacity. In either case, such cases lead to drastic resource wastage which comprises the main Achilles Heel in WSANs due to the typical resource limitations [1]. The average overutilization percentage of all actor nodes is around $-58 \%$ for all the Voronoi types, while the average underutilization is around $+48 \%$ for all the Voronoi types. It is also worth noting that the average maximum overutilization and underutilization percentage shows a significant difference between the Voronoi types. More specifically, the average maximum overutilization/underutilization percentages for both the Euclidean and AWV types are approximately $-220 \%$ and $+65 \%$, respectively, while the same metrics for the MWV approach are around $-165 \%$ and $+75 \%$, respectively. Without a doubt all these percentages indicate emphatic misuse of the actors capacity which effectively leads either to cases where an actor is not able to handle the allocated sensors (compromising both the performance and robustness of the network) or to cases where unacceptable resource wastage is recorded.

In Table 1, the number of iterations needed so as to achieve the assignment of all sensor nodes to the actors is presented. As depicted, the selection technique does not affect significantly the number of iterations. The most critical observation is that the MWV approach needs more iterations so as to finalize the allocation scenario.

6.3. Total Actors Capacity Greater Than the Number of Sensor Nodes. Apart from the relative extreme case where demands posed by sensors are equivalent to the capabilities of the network actors, it is critical to evaluate the proposed selection techniques compared to native Voronoi types in more realistic cases, that is, where the network wide capabilities surpass the aggregate demands of sensors. In this way, it is possible to gain valuable insight into how such sensoractor allocation algorithms behave and even more highlight additional advantages of the proposed approaches. Therefore, in this section, it is assumed that the sensors count remains the same (effectively representing the sensors' requirements), that is, 140 , while the aggregate ASC is 220 , that is, $57 \%$ higher.

In this context, Figure 9 offers critical indication depicting the percentile deviation of the average distance between the sensor and the correlated actor. As it can be easily

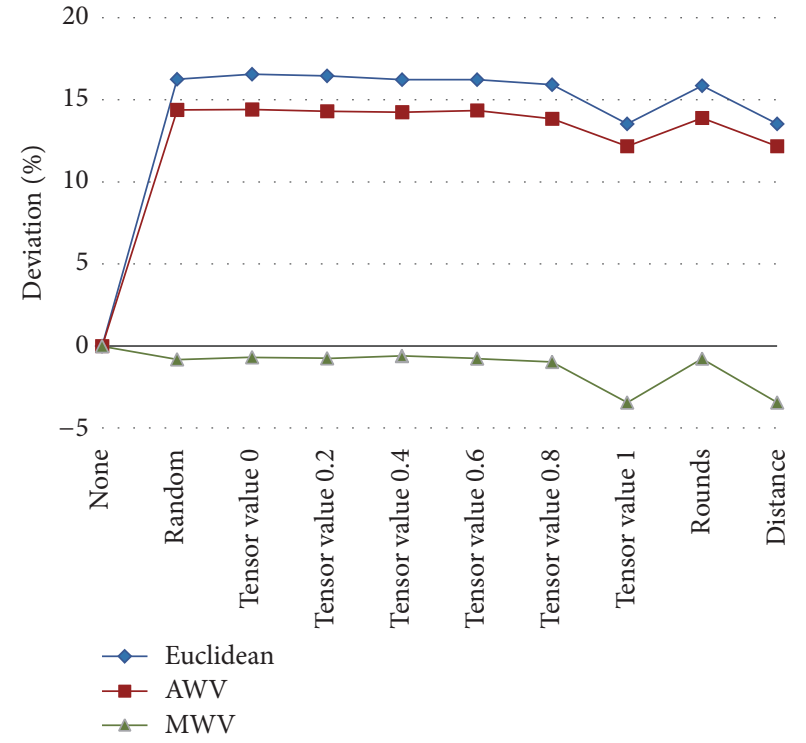

FIGURE 9: Deviation in average distance. Total capacity $=220$.

observed, the application of the selection techniques on the Euclidean and AWV types leads to an increase of the average distance, equal to $17 \%$ and $14 \%$, respectively. Such increase is expected since it effectively represents the tradeoff for optimum actor capacity utilization. However, it is important to indicate that the aforementioned percentages are approximately $50 \%$ reduced compared to the case where capacity is equal to the sensor demands. In other words, the negative aspect of selection techniques utilization is significantly degraded in realistic scenarios, thus advocating the use of the proposed techniques. Furthermore, respective advocacy is emphatically enhanced when considering the MWV Voronoi type. In this case, it is indicated that the application of the selection techniques does not negatively affect the average distance between sensors and correlated actor but in fact it positively affects it, significantly reducing it up to $4 \%$ compared to native MWV approach. Therefore, in such cases, sensor distribution among actors is accompanied by distance reduction, leading to potentially great benefits in energy consumption, communication performance, and network robustness.

Such behavior can be attributed to the nature of MWV algorithms, where the weighted distance can lead to nonconvex Voronoi regions and shows that the application of these techniques optimizes the native MWV performance in terms of the average distance. Finally, it is noted that in all three cases the tensor value variations offer small differences. The deviation in average distance never exceeds $4 \%$ for tensor 


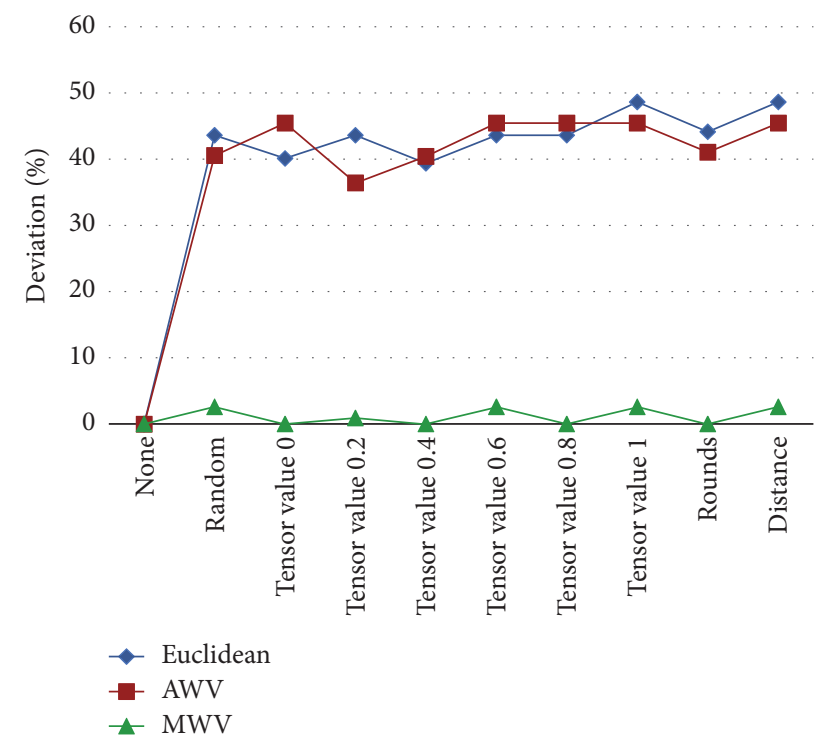

FIGURE 10: Deviation in max distance. Total capacity $=220$.

value equal to one compared to other tensor values $(0.2$, 0.4, 0.6, and 0.8), considering the MWV type. In the other two Voronoi types (Euclidean and AWV), the deviation in average distance never exceeds $2 \%$ for tensor value equal to one compared to the other tensor values $(0.2,0.4,0.6$, and $0.8)$.

Moving on to Figure 10, respective percentile deviation of averaged maximum distances between native Voronoi types (i.e., None technique) and proposed selection-based enhancements are presented. Once again Euclidean and AWV types exhibit considerable increase of final distances between correlated nodes (sensors-actors). However, they are significantly lower (10-20 percentile units) compared to the scenario of equal actor capacity to sensors demands. Also in this case the most important observations concern the MWV case where imposed distance overhead is approximately zero. This observation, in combination with the deviation on the average distance on the MWV, further validates the suitability of applying the proposed selection techniques at this type.

Based on the previous figures, it is easily extracted that a critical goal of selection techniques is to retain imposed distance overhead at it is absolute minimum (although it was observed that in some they even slightly improved it). However, once again the most important aspect is to quantify the improvement that selection techniques offer to the ASC utilization. In that respect, Figure 11 depicts the underutilization and overutilization percentages of the actors when no selection technique is applied. It is critical to emphasize that even in cases where offered capacity is more than $50 \%$ higher than respective demands, native Voronoi types lead to significant misuse of underlying resources (i.e., actors overutilization or underutilization). For example, as shown, although aggregate capacity overpasses respective demands, still average actor overutilization exceeds 50\%, while maximum overutilization can reach up to $200 \%$.

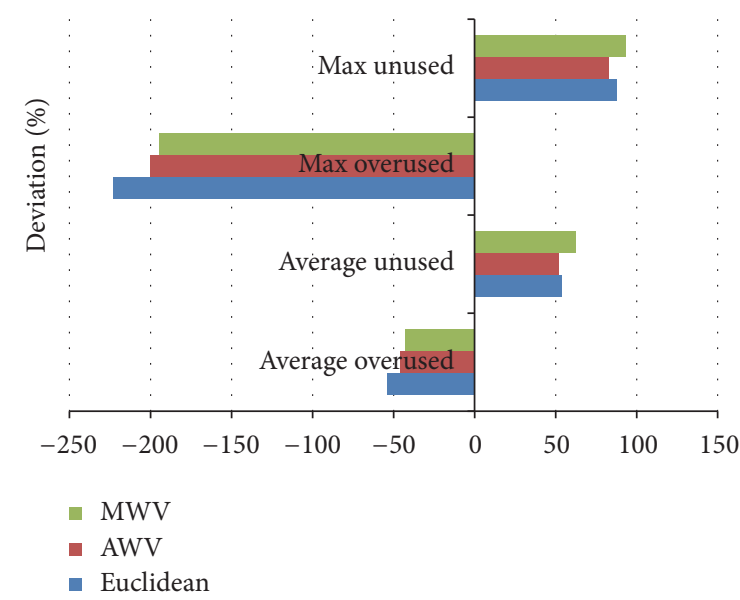

FIgURE 11: Actors utilization with no selection technique. Total capacity $=220$.

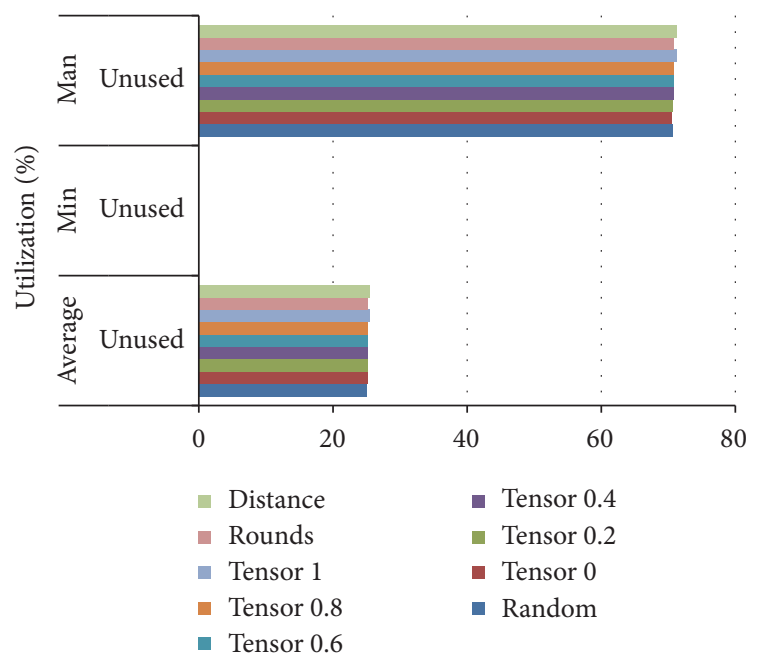

FIGURE 12: Actors utilization: Voronoi type, Euclidean. Total capacity $=220$.

In contrast to such performance, when any of the proposed selection techniques is utilized respective overutilization percentages are zero $(0 \%)$. Of course in this case where capacity exceeds demands, underutilization is by definition expected; therefore respective measurements must be studied carefully in order to extract objective conclusions. In this case, proposed techniques are evaluated with respect to fairness. Specifically, the ideal case would be actors to record approximately $63 \%$ utilization (since $0.63 * 220=140$ ). That would indicate fair allocation of resources to the sensor nodes. Otherwise, cases exist where actors are more intensely used (not necessarily meaning overutilization), thus leading to the potential danger of reduced lifetime or otherwise resource unfair expenditure. Based on the aforementioned analysis and on Figures 12 and 13, the MWV approach offers the optimum approximation to the ideal condition. More specifically, the average underutilized actors' percentage considering the Euclidean approach (for all selection techniques) 
TABLE 2: Average iterations. Total ASC $=220$.

\begin{tabular}{lcccccccccc}
\hline & None & Random & Tensor 0 & Tensor 0.2 & Tensor 0.4 & Tensor 0.6 & Tensor 0.8 & Tensor 1 & Rounds & Distance \\
\hline Euclidean & 0 & 2.52 & 2.53 & 2.52 & 2.51 & 2.51 & 2.54 & 2.54 & 2.56 & 2.54 \\
AWV & 0 & 2.37 & 2.35 & 2.4 & 2.41 & 2.34 & 2.36 & 2.42 & 2.36 & 2.42 \\
MWV & 0 & 1.79 & 1.8 & 1.8 & 1.79 & 1.79 & 1.8 & 1.86 & 1.8 & 1.86 \\
\hline
\end{tabular}

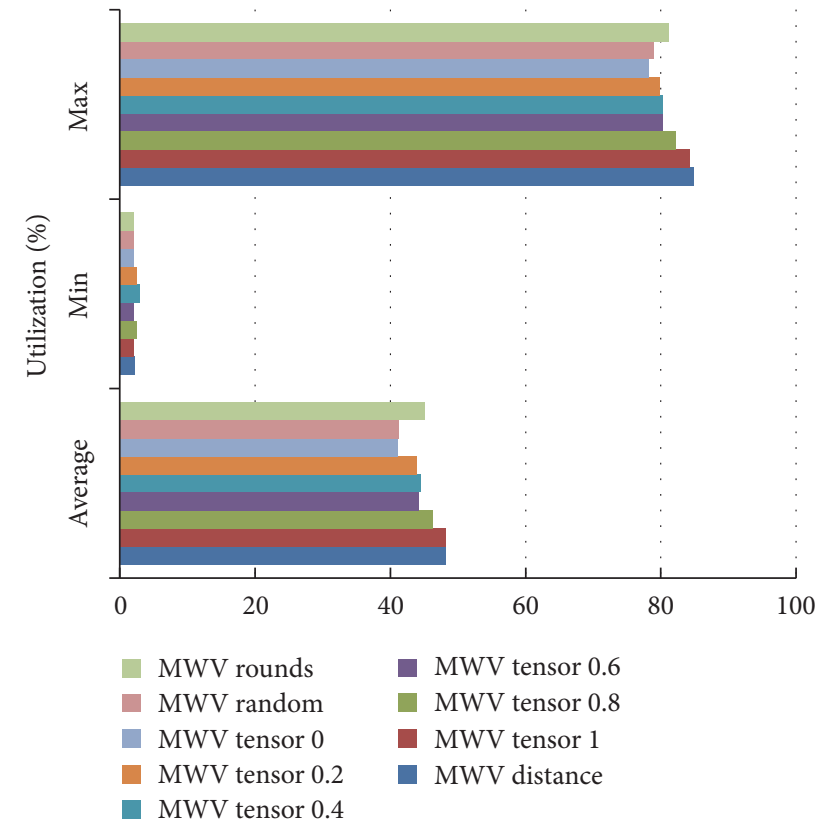

FIGURE 13: Actors utilization: Voronoi type, AWV. Total capacity = 220.

is approximately equal to $27 \%$, while the respective percentage considering the MWV approach varies from $41 \%$ to $46 \%$. As discussed earlier, the ideal utilization percentage is around $63 \%$; thus the underutilization percentage should be around $37 \%$. Hence, the application of the Random and Tensor selection techniques on the MWV type seems to be close enough to the ideal conditions. In other words, MWV approach seems to yield a more fair allocation than the Euclidean approach regarding the actors' resource allocation. This is also explained from the min underutilization percentage which is approximately equal to 0 , which means that there are actors that are fully utilized, while other actors are not considered at all for the final assignment scenario.

In Table 2, the number of the iterations needed for achieving the final assignment is depicted. As it can be observed, the MWV approach seems to be faster than the Euclidean and MWV approaches, as it requires less than two iterations so as to extract the allocation plan.

\section{Conclusions and Future Work}

Wireless Sensor Networks are increasingly utilized in a wide range of applications where sensor-to-actor coordination is of utmost importance. However, despite its criticality, existing efforts do not take into consideration the particular characteristics and requirements of WSNs pertaining to scarce resources and fair allocation of workload according to sensors' capabilities, respectively. Hence, in this work, we propose a novel node-to-actor coordination based on the Voronoi tessellation. The actor's capabilities are represented by the term of the Actor Service Capacity and the main objective of this work is to provide a coordination mechanism that will guarantee that the number of sensors assigned to each actor is lower than/equal to its service capacity. Towards this objective, a set of selection techniques that are applied on the Voronoi algorithm is proposed. The comprehensive evaluation analysis, on the proposed algorithm, highlighted the importance of the proposed extension by integrating selection techniques in order to effectively tackle the problem of unfair actors' resources allocation. More specifically, in case that the total ASC is equal to the number of nodes, the application of the proposed algorithm leads to zero overutilization percentage for all the Voronoi types, while for the same cases the native approaches lead to $58 \%$ and $48 \%$ overutilization and underutilization percentages corresponding to emphatic resource wastage and network degraded performance. When considering the total ASC greater than the number of nodes, the average overutilization percentage by the native approaches is around 50\%, while the application of a selection technique once again assures $0 \%$ overutilization. In addition, considering the case where the total capacity is greater than the number of nodes, the application of a proposed selection technique on the MWV type leads to a $4 \%$ reduction of the average distance compared to the native approach ("None"). Finally, the application of the Random and Tensor techniques on the MWV type yields a more fair assignment scenario compared to the Euclidean and AWV types, which also highlight the suitability of the application of the proposed selection techniques.

We believe that this work is the first step of the proposed sensor-to-actor evaluation analysis, proving the required proof of concept concerning the significant advantages of proposed solutions taking into consideration specific WSN characteristics. Driven by the validation and promising results this paper offers, the proposed approach will be extended towards the following directions: (i) the implementation and the porting of this sensor-actor coordination algorithm in a wireless actor platform, (ii) the implementation of a control protocol so as to enable the intercommunication between the sensor and the actor nodes in order to inform the sensor nodes about the actor they have been assigned to, taking into account sensors, connectivity characteristics, and actors' range capabilities, (iii) the simulated evaluation analysis of the aforementioned features, considering periodic execution of the sensor-actor coordination algorithm while changing ASC, (iv) the simulated evaluation analysis of 
the aforementioned features, considering periodic execution of the sensor-actor coordination algorithm while changing actor's location, which can lead to network partitioning, and (v) a real hardware evaluation analysis.

\section{Conflicts of Interest}

The authors declare that there are no conflicts of interest regarding the publication of this paper.

\section{References}

[1] I. F. Akyildiz and I. H. Kasimoglu, "Wireless sensor and actor networks: research challenges," Ad Hoc Networks, vol. 2, no. 4, pp. 351-367, 2004.

[2] F. Khan, S. Khan, and S. A. Khan, "Performance improvement in wireless sensor and actor networks based on actor repositioning," in Proceedings of the International Conference on Connected Vehicles and Expo, ICCVE 2015, pp. 134-139, Shenzhen, China, 2015.

[3] M. Kamali, L. Laibinis, L. Petre, and K. Sere, "Self-recovering sensor-actor networks," in Proceedings of the 9th International Workshop on the Foundations of Coordination Languages and Software Architectures (FOCLASA '10), M. Mousavi and G. Salaün, Eds., vol. 30 of Electronic Proceedings in Theoretical Computer Science, pp. 47-61, EPTCS, Paris, France, 2010.

[4] M. Imran, A. M. Said, M. Younis, and H. Hasbullah, "Application-centric connectivity restoration algorithm for wireless sensor and actor networks," in Proceedings of the 6th International Conference, GPC 2011, J. Riekki, M. Ylianttila, and M. Guo, Eds., vol. 6646 of Advances in Grid and Pervasive Computing, pp. 243-253, Springer, Oulu, Finland, 2011.

[5] F.-J. Wu, Y.-F. Kao, and Y.-C. Tseng, "From wireless sensor networks towards cyber physical systems," Pervasive and Mobile Computing, vol. 7, no. 4, pp. 397-413, 2011.

[6] E. Ruiz-Ibarra and L. Villasenor-Gonzalez, "Cooperation mechanism taxonomy for wireless sensor and actor networks," in Proceedings of the IFIP Conference on Wireless Sensor and Actor Networks (WSAN '08), A. Miri, Ed., vol. 264 of Wireless Sensor and Actor Networks II, pp. 62-73, Springer, Ontario, Canada, 2008.

[7] S. M. Dima, C. Panagiotou, D. Tsitsipis, C. Antonopoulos, J. Gialelis, and S. Koubias, "Performance evaluation of a WSN system for distributed event detection using fuzzy logic," Ad Hoc Networks, vol. 23, pp. 87-108, 2014.

[8] F. Dressler, Self-Organization in Sensor And Actor Networks, John Wiley \& Sons, 2008.

[9] T. Melodia, D. Pompili, V. C. Gungor, and I. F. Akyildiz, "A distributed coordination framework for wireless sensor and actor networks," in Proceedings of the 6th ACM International Symposium on Mobile Ad Hoc Networking and Computing, MobiHoc '05, pp. 99-110, New York, NY, USA, 2005.

[10] J. Long, C. Gao, S. He, X. Liu, and A. Liu, "Bridging the gap among actor-sensor-actor communication through load balancing multi-path routing," EURASIP Journal on Wireless Communications and Networking, vol. 2015, no. 1, article 256, pp. 122, 2015.

[11] M. I. Akbaş and D. Turgut, "Lightweight routing with dynamic interests in wireless sensor and actor networks," Ad Hoc Networks, vol. 11, no. 8, pp. 2313-2328, 2013.
[12] V. C. Gungor, O. B. Akan, and I. F. Akyildiz, "A real-time and Reliable Transport (RT) ${ }^{2}$ protocol for wireless sensor and actor networks," The IEEE/ACM Transactions on Networking's, vol. 16, no. 2, pp. 359-370, 2008.

[13] J. Chen, M. Díaz, L. Llopis, B. Rubio, and J. M. Troya, "A survey on quality of service support in wireless sensor and actor networks: requirements and challenges in the context of critical infrastructure protection," Journal of Network and Computer Applications, vol. 34, no. 4, pp. 1225-1239, 2011, Advanced Topics in Cloud Computing.

[14] F. Bouhafs, M. Merabti, and H. Mokhtar, "A coordination protocol for wireless sensor and actor networks," vol. 1, 2006.

[15] T. Melodia, D. Pompili, and I. F. Akyildiz, "A communication architecture for mobile wireless sensor and actor networks," in Proceedings of the 3rd Annual IEEE Communications Society on Sensor and Ad hoc Communications and Networks, Secon 2006, vol. 1, pp. 109-118, Reston, Va, USA, 2006.

[16] S. Beheshti, M. G. Farahani, M. Sharifnejad, and M. Sharifi, "An enhanced routing protocol for wireless sensor networks," International Journal of Electronics Communication and Computer Engineering, vol. 5, pp. 1371-1374, 2011.

[17] K. Akkaya, F. Senel, and B. McLaughlan, "Clustering of wireless sensor and actor networks based on sensor distribution and connectivity," Journal of Parallel and Distributed Computing, vol. 69, no. 6, pp. 573-587, 2009.

[18] M. Okhovvat, M. Sharifi, and H. Momeni, "Task allocation to actors in wireless sensor actor networks: an energy and time aware technique," Procedia Computer Science, vol. 3, pp. 484490, 2011.

[19] A. Ghosh, N. Mangalvedhe, R. Ratasuk et al., "Heterogeneous cellular networks: from theory to practice," IEEE Communications Magazine, vol. 50, no. 6, pp. 54-64, 2012.

[20] Z. Dai, B. Wang, Z. Li, and A. Yin, "VDSPT: a sensor-actor coordination protocol for wireless sensor and actor network based on voronoi diagram and shortest path tree," in Proceedings of 1st International Symposium on Computer Network and Multimedia Technology, (CNMT '09), pp. 1-4, Wuhan, China, 2009.

[21] F. Aurenhammer, "Voronoi diagrams-a survey of a fundamental geometric data structure," ACM Computing Surveys, vol. 23, no. 3, pp. 345-405, 1991.

[22] M. A. M. Viera, L. F. M. Viera, L. B. Ruiz, A. A. F. Loureiro, A. O. Fernandes, and J. M. S. Nogueira, "Scheduling nodes in wireless sensor networks: a Voronoi approach," in Proceedings of the 28th Annual IEEE International Conference on Local Computer Networks, LCN 2003, Held in conjunction with the Workshop on High-Speed Local Networks, HSLN 2003 and Workshop on Wireless Local Networks, WLN 2003, pp. 423-429, Bonn, Germany, 2003.

[23] J. Li, R.-C. Wang, H.-P. Huang, and L.-J. Sun, "Voronoi based area coverage optimization for directional sensor networks," in Proceedings of the 2nd International Symposium on Electronic Commerce and Security, (ISECS '09), pp. 488-493, Nanchang , China, 2009.

[24] J. Stergiopoulos and A. Tzes, "Convex Voronoi space-partitioning for coverage purposes in heterogeneous sensor networks," in Proceedings of the 10th European Control Conference, ECC 2009, pp. 2361-2366, Hungary, 2009.

[25] S. Nithyakalyani and S. Suresh Kumar, "Energy efficient data aggregation using voronoi based genetic clustering algorithm in WSN," International Journal of Computer Applications, vol. 54, no. 4, pp. 37-41, 2012. 
[26] M. Imran, A. M. Said, M. Younis, and H. Hasbullah, "Application-centric recovery algorithm for wireless sensor and actor networks," International Journal of Communication Networks and Distributed Systems, vol. 10, no. 4, pp. 379-401, 2013.

[27] N. Haider, M. Imran, M. Younis, N. Saad, and M. Guizani, “A novel mechanism for restoring actor connected coverage in wireless sensor and actor networks," in Proceedings of the IEEE International Conference on Communications, ICC 2015, pp. 6383-6388, London, UK, 2015.

[28] T. Melodia, D. Pompili, V. C. Gungor, and I. F. Akyildiz, "Communication and coordination in wireless sensor and actor networks," IEEE Transactions on Mobile Computing, vol. 6, no. 10, pp. 1116-1129, 2007.

[29] H. Nakayama, Z. M. Fadlullah, N. Ansari, and N. Kato, "A novel scheme for WSAN sink mobility based on clustering and set packing techniques," IEEE Transactions on Automatic Control, vol. 56, no. 10, pp. 2381-2389, 2011.

[30] D. Elmazi, E. Kulla, T. Oda, E. Spaho, S. Sakamoto, and L. Barolli, "A comparison study of two fuzzy-based systems for selection of actor node in wireless sensor actor networks," Journal of Ambient Intelligence and Humanized Computing, vol. 6, no. 5, pp. 635-645, 2015.

[31] A. Breitenmoser, M. Schwager, J.-C. Metzger, R. Siegwart, and D. Rus, "Voronoi coverage of non-convex environments with a group of networked robots," in Proceedings of the IEEE International Conference on Robotics and Automation (ICRA '10), pp. 4982-4989, IEEE, Anchorage, Alaska, USA, 2010.

[32] M. I. Karavelas and I. Z. Emiris, "Root comparison techniques applied to computing the additively weighted Voronoi diagram," in Proceedings of the 14th Annual ACM-SIAM Symposium on Discrete Algorithms, SODA '03, pp. 320-329, Society for Industrial and Applied Mathematics, Baltimore, Md, USA, 2003.

[33] R. Mulligan and H. M. Ammari, "Coverage in wireless sensor networks: a survey," Network Protocols and Algorithms, vol. 2, no. 2 , pp. $27-53,2010$. 


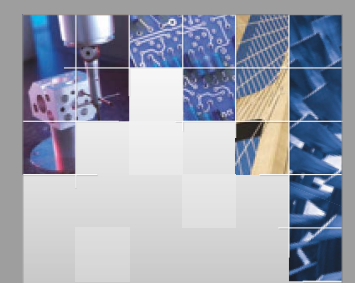

\section{Enfincering}
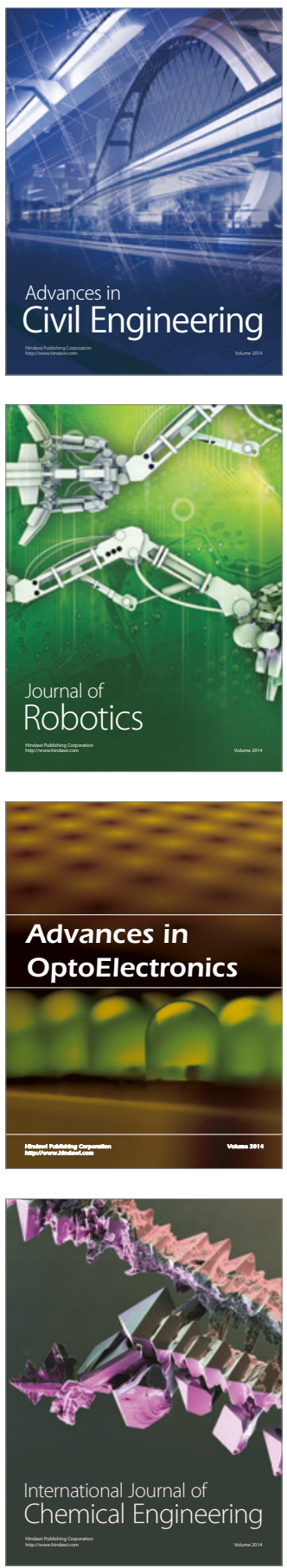

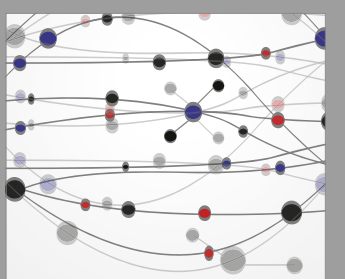

The Scientific World Journal

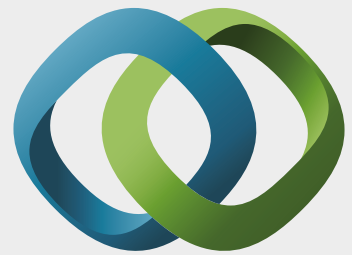

\section{Hindawi}

Submit your manuscripts at

https://www.hindawi.com
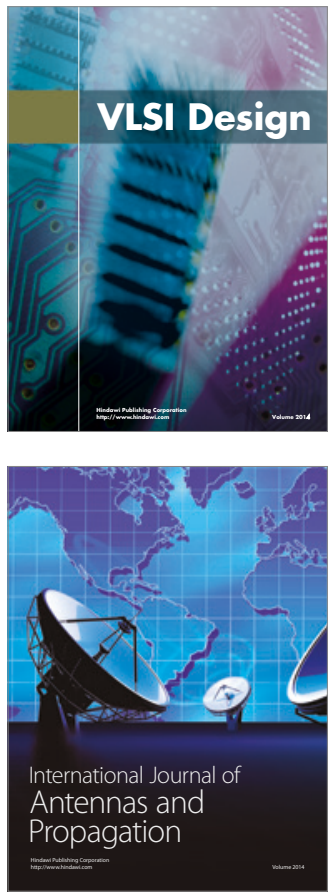

\section{Rotating}

Machinery
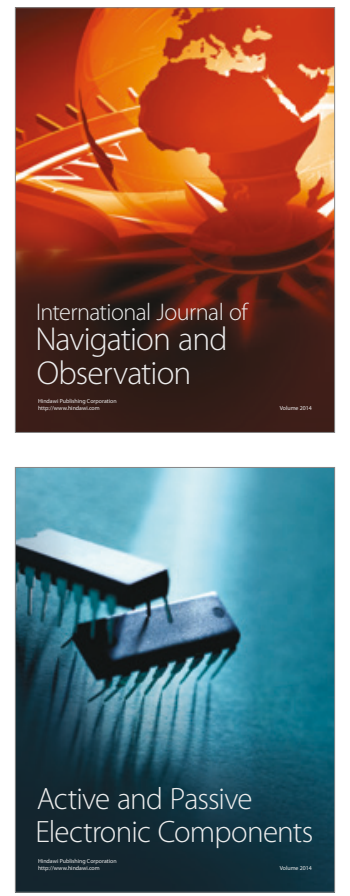
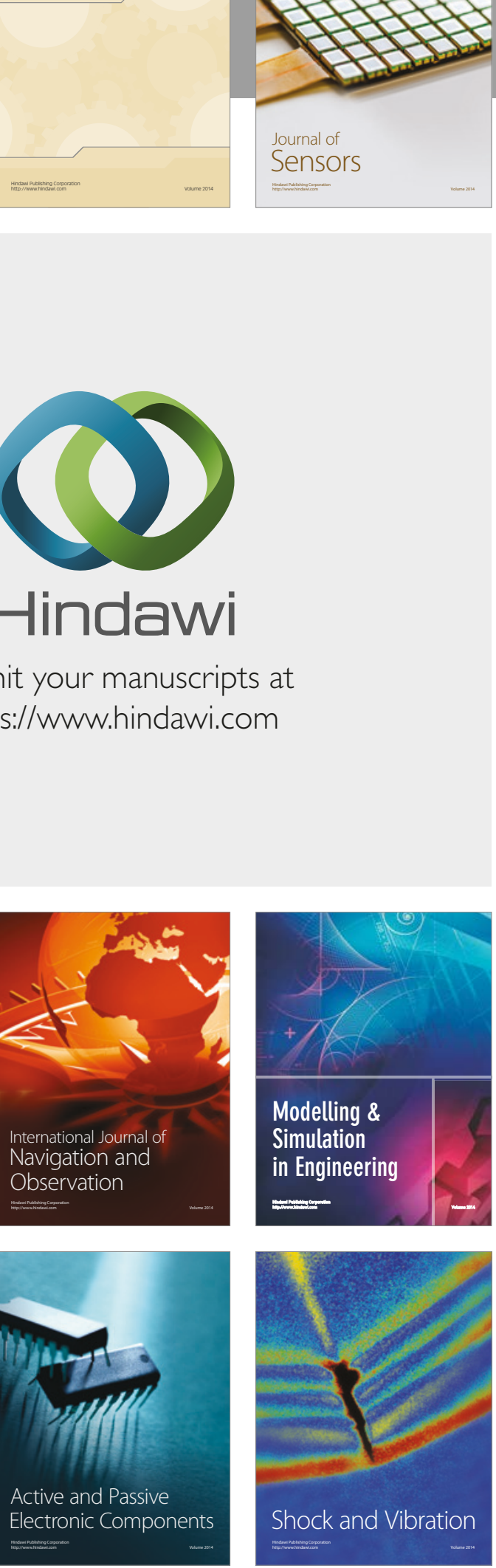
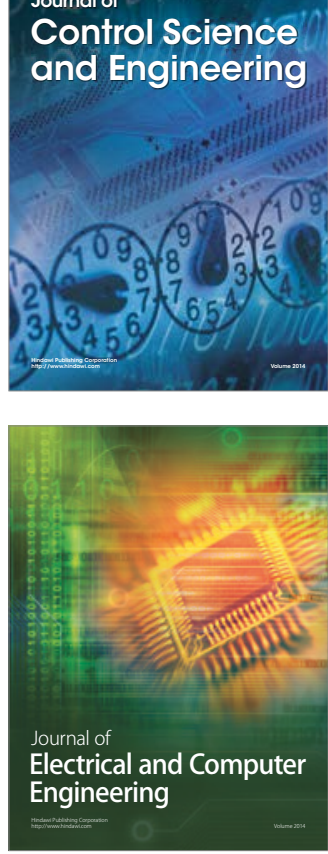

Distributed

Journal of

Control Science

and Engineering
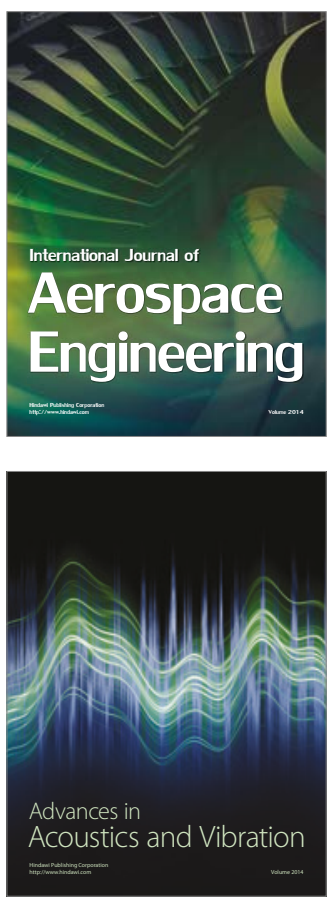

Sensor Networks 